\title{
GLACIOLOGY AND GEODYNAMICS
}

\author{
By R. F. KING \\ (Department of Geology, Birmingham University)
}

A LARGE body of geological evidence about the Ice Ages of the Pleistocene exists, indicating a pattern of major glacial and inter-glacial periods, minor advances and withdrawals of ice cover and associated climatic fluctuations. This has been extensively studied. It is only recently, however, that the time-scale of this pattern has become established, and even now there seems room for dispute about such major questions as the contemporaneity of the Pleistocene glaciations in different parts of the globe.

Lack of information has never deterred geophysical speculation, and the past existence of ice caps much greater than those known to-day clearly calls for an explanation, particularly since in the more remote past, these ice caps appear to have been formed in what are now temperate or subtropical regions. At present, one school of thought holds that world-wide climatic fluctuations due to changes in the intensity of the solar radiation must have been responsible for the ice ages of the past. The other school, with which we are concerned in this review, takes the uniformitarian view that the climatic zones have always, on average, been as they are to-day. The glaciations are then explained by supposing that the region in question was situated at one of the poles in the past. The adherents of this school, then, postulate movement of the continental masses relative to the Earth's pole of rotation ("polar wandering") and sometimes also relative to each other ("continental drift"), and so enter the field of geodynamics, which is concerned, among other things, with the problems of the stability of the pole and the mobility of continents. Violent controversies have centred on these problems for many years, since many (though not all) geologists have held that they are confronted with evidence inconsistent with the present positions of the continents, whilst geophysicists have been practically unanimous in asserting that both polar wandering and continental drift are dynamically impossible. The controversies have recently been given an added spice by the appearance of geophysicists in alliance with the geological faction, as a result of the revival of interest in palaeomagnetism, with its indications of large polar movements and probably also of relative movements of the continents. The evidence from measurements on the remanent magnetization of rocks in Europe suggests that the pole moved into its present position in late Tertiary times, after tracing a path across the Pacific and along the east coast of Asia at an average rate of about one-third of a degree in a million years. This polar path is broadly consistent with that required on palaeoclimatological grounds, and this is also true for polar paths which have been calculated for other continents. The polar paths for different continents are not identical, however, and this is held to indicate that continental drift, as well as polar wandering, has occurred.

Mr. Karl A. Pauly, in his Cause of the great Ice Ages* (privately printed, Schenectady, I957) postulates a very different type of polar wandering. He believes that the Pleistocene glaciations proceeded from seven consecutive centres, and places the pole at each of these centres in turn, at distances of the order of 30 degrees from the present pole and from each other. He calculates the "Pleistocene" latitude of 27 terminal moraines of Pleistocene mountain glaciers, well distributed over the world, assuming that each was formed during the nearest approach of the pole. He thus obtains a curve relating this latitude to their elevation above present sea level. This curve is said to be identical with that plotted for 17 modern terminal moraines. The agreement is indeed good, but the scatter of the points is very considerable, and one suspects that proponents of the "climatic fluctuation" group of

* Further details of the works reviewed in this article will be found in Ice, Nos. 2 and 3, January and July 1958. 
theories could claim an equally smooth relationship between elevation and "modern" latitude for the Pleistocene moraines. Pauly does in fact plot the points for such a graph but omits to draw a curve through them.

The second part of the book is devoted to Pauly's theory of the mechanics of polar movement. Like all advocates of polar wandering, he admits that an appreciable movement of the Earth's axis in space is impossible, but instead supposes that the lithosphere as a whole must slide over the mantle. He ignores the rather more plausible model of Gold ${ }^{\mathrm{I}}$ in which the earth as a whole moves relative to its rotational axis, whilst the mantle deforms plastically in such a way as to annul the separation of the axes of rotation and figure. It seems to the present writer that Pauly's explanation of the mechanics of crustal displacements is based on an incomplete understanding of Eddington's tentative suggestions ${ }^{2}$ about the role of tidal forces, and is unlikely to find acceptance.

Dr. C. H. Hapgood has elaborated the hypothesis of polar wandering to a much greater extent than has Mr. Pauly. In Earth's shifting crust (New York, Pantheon Books, 1958) he is led to postulate that during the Würm glaciation the pole was situated near Hudson Bayagain about 30 degrees from its present position-and that the eccentric ice cap formed there provided a centrifugal force which drove the lithosphere over the mantle to its present position. He investigates the consequences of this hypothesis and finds that they are at the worst not inconsistent with the facts. Encouraged by this, he places earlier pole positions somewhat more tentatively in Greenland and Alaska: this sequence of poles by no means agrees with Pauly's, whom he nevertheless quotes with approval. It should also be placed on record that Hapgood's quotation (p. 308) of the magnetic evidence from Japan (Nagata ${ }^{3}$ ) is in fact a misquotation.

The great value of this book lies in the author's collection of evidence in favour of polar shifts: whether we believe in his particular conception of them or disbelieve in them entirely, this evidence must be seriously considered. Hapgood is to be congratulated on the explicitness of his statement of his theory, which at least provides a clear basis for argument. Briefly, the theory is that major displacements of the lithosphere have always occurred from time to time and that they are always caused by eccentric near-polar ice caps, supposed (somewhat contrary to existing evidence) not to be isostatically compensated. The time-scale of the movements is given by the requirement that the pole is supposed to have been in Labrador until about 10,000 years ago. This gives a rate of polar movement of about one-third of a degree in a century, ten thousand times faster than that indicated by rock magnetism.

The termination of a polar movement would naturally be brought about by the melting of the motive ice cap as it reached temperate latitudes, and the pole is then supposed to remain fixed whilst a new ice cap is built up, until the centrifugal stresses again suffice to slide the lithosphere on the mantle. The geodynamics of this process have been worked out for Dr. Hapgood by Mr. James H. Campbell, who is, like Mr. Pauly, an engineer. Campbell supposes that an "asthenosphere" of negligible shear strength and viscosity exists beneath the crust (this assumption for times of the order of 10,000 years seems to be of very doubtful validity) and that therefore the only forces opposing the tendency of an unbalanced ice cap to move to the equator are those due to the tailoring of the lithosphere to fit the Earth's equatorial bulge. When the centrifugal force reaches a certain critical limit, the crust splits down the seams and turns on the mantle about an equatorial axis. As it does so, alternate quadrants of the crust will be stretched (with resulting subsidence) and compressed (and so elevated). Hapgood follows up the implications of this model and attempts to compare the fracture and stress patterns predicted by Campbell with those actually observed. Although the case is well presented, with no more bias than one would expect from a true enthusiast, it is an attractive rather than a convincing one. Perhaps this is why the author has addressed it to a lay audience, and has thought it necessary to adduce the support of Einstein (in a Foreword) for his views rather than that of an eminent geologist or geophysicist. I't would be 
unfortunate if, for this reason, glaciologists and other geophysicists should not read the book, for it is indeed a most stimulating one.

Dr. Hapgood and Mr. Pauly both have geophysical axes to grind, being anxious to convince their readers of the truth of their ideas: Dr. A. E. Scheidegger, whose Principles of geodynamics (Berlin, Springer Verlag, 1958) has also appeared recently, has played the advocate in the past but now claims to have reached a state of geophysical agnosticism. His book is a most valuable compilation of the various theories of the dynamics of the Earth, with particular emphasis on those relating to orogenesis.

The book begins with a strictly factual, though necessarily very brief, account of the physiography of the Earth, and continues with another short chapter on the geophysical data of seismology, gravity measurements, etc. The third chapter is on the flow and fracture of matter in general and the Earth in particular, and emphasizes our ignorance of the exact rheological behaviour of the Earth. The core of the book consists of three chapters on the Earth's Rotation, Continents and Oceans, and Orogeneses, in which the author substantiates his claim of agnosticism by presenting a number of geodynamic theories and finding at least one serious objection to each of them. Finally, the dynamics of faulting, folding and other smaller-scale phenomena are considered, including the post-glacial uplift of Fennoscandia.

The problem of polar wandering is treated in this book only from the point of view of movements of the Earth as a whole: rapid relative movements of lithosphere and mantle as envisaged by Hapgood and Pauly are not even considered. It seems that a rate of polar wander but little greater than that indicated by rock magnetism is geodynamically permissible. As for continental drift, which, strangely enough, Hapgood dismisses as impossible, Scheidegger finds this the least unsatisfactory of the many theories of orogenesis which he considers.

Dr. Scheidegger's book is a valuable addition to geophysical literature. It is well presented and printed, and a pleasure to read, and one cannot but hope that his next book may be an advance from agnosticism to a geophysical faith, based perhaps on his own contributions to the science, which will prove more viable than those he reviews.

\section{MS. received 20 October $195^{\circ}$}

\section{REFERENCES}

1. Gold, T. Instability of the Earth's axis of rotation. Nature, Vol. I 75, No. 4456, I955, p. 526-29.

2. Eddington, A. S. The borderland of geology and astronomy. Nature, Vol. I I I, No. 2775, 1923, p. 20-2 I.

3. Nagata, T., and others. Palaeomagnetic study on a Quaternary volcanic region in Japan. Advances in Physics, Vol. 6, No. 23, 1957, p. 255-63.

\section{REVIEW}

\section{THE QUATERnary ERA.* J. K. Charlesworth. London, Arnold.}

The word "masterpiece" is often misused and it may seem rather odd to apply it to a publication which is by its very nature based almost wholly on the work of others, yet it seems a highly appropriate term for these two volumes. In scientific publications there is an increasing gap between the one-man text-book and the comprehensive summary of a major field of knowledge carried out as a team project. Professor Charlesworth has attempted and brilliantly achieved a task on the latter scale by a magnificent personal effort spread over 35 years.

The first volume is in two parts. Part I, Glaciology, deals principally with 'Land Ice', but with a valuable section covering the increasingly topical 'Sea Ice'. Snow and its dis-

\footnotetext{
* A short notice of this work appeared in Ice, No. 2, July 1958, p. ro-I I.
} 